\title{
Swiss Cheese, Drosophila Ortholog of Hereditary Spastic Paraplegia Gene NTE, Maintains Neuromuscular Junction Development and Microtubule Network
}

\author{
Elena Ryabova, Nataliya Matiytsiv, Olena Trush, \\ Iryna Mohylyak, Galina Kislik, Pavel Melentev and \\ Svetlana Sarantseva
}

Additional information is available at the end of the chapter

http://dx.doi.org/10.5772/intechopen.73077

\begin{abstract}
Neuropathy target esterase (NTE) is a molecular target for the organophosphorus compound-induced delayed neuropathy (OPIDN) and also one of the genetic factors responsible for the development of the hereditary spastic paraplegia (HSP), characterized by axon degeneration of motoneurons causing progressive lower-limb spastic paralysis. Both HSP and OPIDN are characterized by the distal axonopathy. The molecular mechanisms underlying the axonopathy involved in HSP and OPIDN are poorly understood. In order to have a better understanding of the mechanisms that NTE is involved in, we used one of the homologs, human NTE. Swiss cheese (sws) is a Drosophila melanogaster ortholog of NTE with 39\% homology. Mutations in sws as it was shown before lead to age-dependent neurodegeneration, structure alteration of glia cells, and reduced insect life span. To study SWS functions, we used the system of the third-instar larval neuromuscular junctions of $D$. melanogaster. In this study, we show that mutations in sws (sws ${ }^{1}$ and $s w s^{76-1}$ ) and SWS knockdown alter neuromuscular junction's morphology and synaptic microtubules organization.
\end{abstract}

Keywords: axon degeneration, Drosophila melanogaster, neuromuscular junctions, neuropathy target esterase (NTE), Swiss cheese (sws)

\section{Introduction}

It has recently been established that mutations in the particular region of the neuropathy target esterase (NTE) gene coding for the catalytic domain of the NTE protein cause an 
autosomal-recessive form of HSP (SPG39) Gordon-Holmes syndrome, Boucher-Neuhäuser syndrome, Laurence-Moon syndrome, Oliver-McFarlane syndrome, and Leber's congenital amarosis [1-4]. Initially, NTE was found in human brain homogenates as an enzyme, the activity of which could be inhibited by organophosphates, leading to the development of organophosphorus compound-induced delayed neuropathy (OPIDN) [5]. HSP and OPIDN are both characterized by the distal degeneration of motor and sensory axons [6, 7].

NTE is a highly conserved protein with homology among many organisms (from yeast to humans), particularly in its esterase catalytic domain [8]. The NTE ortholog in Drosophila melanogaster (D. melanogaster, Meigen, 1830) is called Swiss cheese (sws). All the currently known sws mutants were obtained and described by Kretzschmar et al. [9]. These mutants developed axonal and glial pathology in the brain and neuronal apoptosis [9]. The level of phosphatidylcholine was increased in the mutants [10]. SWS and NTE share high structural (39\%) and functional homology [10]. These proteins are widely expressed in the nervous system [8, 10], localized on the endoplasmic reticulum (ER), and also considered to have esterase activity [11, 10]. NTE knockout mice (mouse and human NTE genes have $95 \%$ homology) die on the ninth day of the embryonic development, unlike mice without NTE expression only in neurons. Mice without NTE neuronal expression develop a phenotype similar to sws mutants, including vacuolization, myelin production, and neuronal death [11]. NTE knockdown in zebra fish leads to development defects, axon shortening, and reduction of axonal arborization [12]. There have been many studies dedicated to NTE/sws; however, we still do not know much about their exact roles in the development and functioning of the nervous system.

In our study, we identified a new allele in sws using genome screening. We also carried out functional studies of sws in vivo, using larval neuromuscular junctions (NMJs) of D. melanogaster as a good system of HSP modeling. We showed that sws is widely expressed in the larval nervous system, especially in glial cells. We also established that mutations in the sws gene alter NMJ morphology, the distribution of synaptic markers, microtubule (MT) network, and synaptic microtubules organization.

\section{The new sws allele}

During the massive screen tests searching for $\mathrm{X}$-linked mutants with age-dependent neurodegeneration, we analyzed paraffin-embedded histological sections of the brain tissue in mutant flies with life span reduction [13]. Sections from a number of mutants showed strong brain vacuolization similar to sws phenotype described by Kretzschmar et al. [9]. However, in the $s w s^{76-15}$ line, we revealed a different phenotype with small vacuoles in all brain regions, which is not a hallmark of sws [13] (Figure 1C and $\mathbf{H}$ ). Therefore, we believe that it is a new allele of sws, and we used deficiency mapping on 7D1-D5 band (Df (1) C128) which uncovered a sws phenotype [9]. To confirm localization in this region, we rescued a neurodegenerative phenotype with 7D1 band duplication $\left(\mathrm{Dp}(1 ; 3) \mathrm{sn}^{13 a 1}\right)$. We were successful in mapping the $s w s^{76-15}$ mutant using deficiency. In addition, heterozygous individuals used for complementation analysis with $s w s^{1}\left(s w s^{1} / s w s^{76-15}\right)$ and $s w s^{4}\left(s w s^{4} / s w s^{76-15}\right.$, data not shown) showed a mutant phenotype, which corresponds to $s w s^{76-15}$-like phenotype (Figure 1E and J). We suggested that this allele is neomorphic and dominant to other sws alleles but recessive to the wild type. 

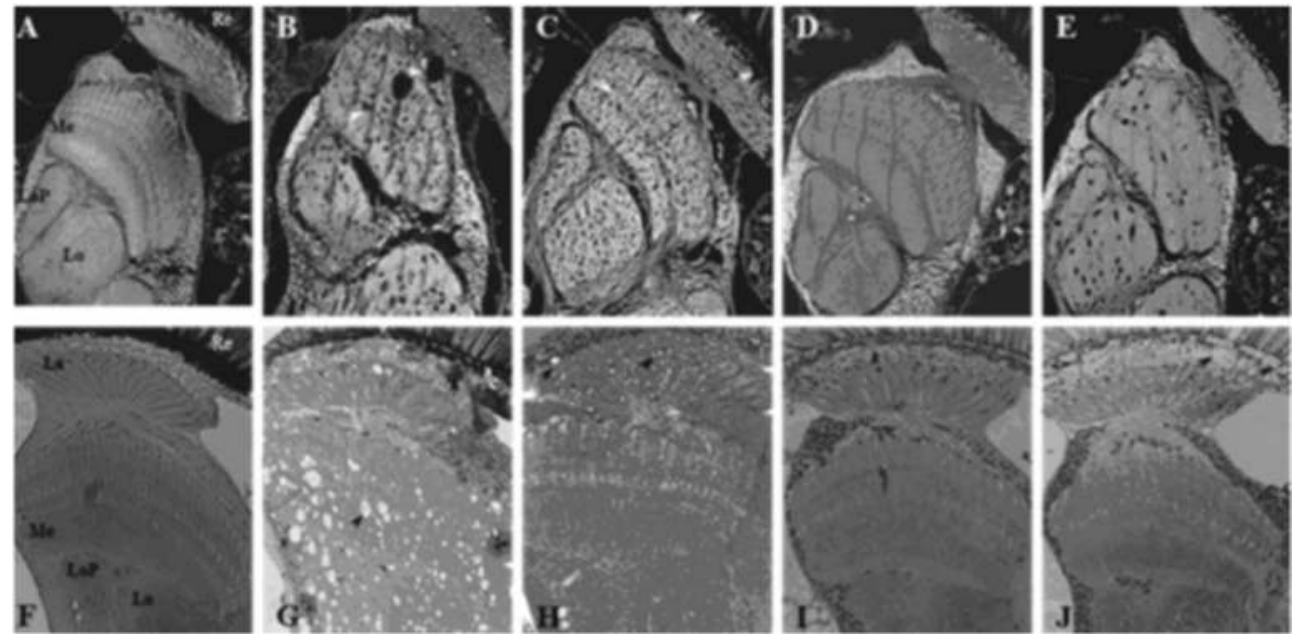

Figure 1. Histological sections of a brain's optic lobe in a 20-day-old Drosophila melanogaster. (A-E) Horizontal 7- $\mu$ m paraffin-embedded sections. (F-J) Horizontal 1- $\mu$ m plastic semi-thin sections. (A and F) Homozygous wild-type tissue of Oregon males used as controls (Bloomington Stock Centre). Neuropile and glia have very regular structure in all layers of the lobe. (B and G) Homozygous sws ${ }^{1}$-mutant males, degeneration tissue is clearly seen as vacuoles in all neuropile and highly stained glia cells hyperwrapping in the lamina cortex. ( $\mathrm{C}$ and $\mathrm{H}$ ) Homozygous $\operatorname{sws}^{76-15}$ males with small vacuoles in neuropile. (D and I) Heterozygous sws ${ }^{1 / O r e g o n}$ old females used as controls in complementation test, glia and neuropile show wild-type phenotype. (E and J) Heterozygous $s w s^{1} / s w s^{76-15}$ old females with mutant phenotypes in neuropile and lamina cortex glia, suggesting mutations in the same gene. Re, retina; La, lamina; Me, medulla; Lo, lobula; LoP, lobula plata. Paraffin-embedded sections were analyzed using a Nikon LSM A1 Clem confocal microscope; plastic-embedded semi-thin sections were analyzed using Karl Zeiss light microscope. Microscopic pictures were taken at the same level of the brain.

\footnotetext{
1 MDVLEMLUSASCSYNTIFSDA WCQYVSKOITATVYMYFALVMMSUFA WFLYFKRMARLRLRDEURSISTVTNSSCDMRGLRFRKRDKMLYYGRRML.100 101 RKMKNVSCQMYSSGKOTKRRAVMRFARRLOLRRDNMPLEMRTVEPAEYLEETECSDRVFPALYMLOSIRIFGHFEKPVFLLCKHTQUELMGDY 200 201 LFKITDPDOSVYIVQSCMINVYISNADGSLSKTVRKGESVTSLLSFIDVLSCNPSYYKTVTAKAIEKSVVIRLPMOAFEVVFONPDVMIRVIQVIMII 300 ivo'

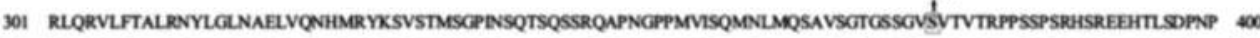
401 NPDCSFHGTTNLTEVHCDAPNADLFOQOQOHSVGNLSTRRSSTLMAPDCHSCLQTTGVTTSIDMRL VQSSAVDSLRKELGLSEEDSHIIEPFVELR S00

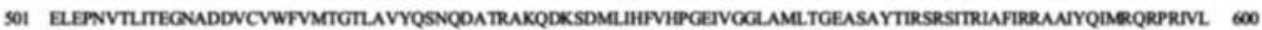
601 DLGNOVVRRLSL VROCDYALDIFLESGRAVYRQDESSDSTYIVLSGRMRSVITHPCCKKEIVGEYGKGDLVGIVEMTETSRTTTVMAVRDSELAKLP T00

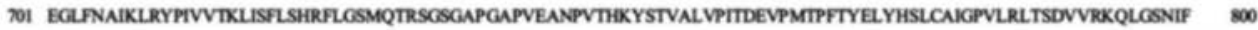

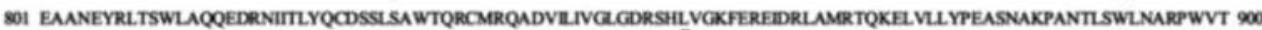
D.

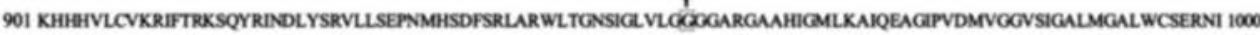
1001 TTVTQKAREWSKKMTKWFLLLDLTYPTSMFSGREFNTHHDTFGDVSIEDL WIPYFTLTTDITASCHRIHTNCSL.WRYVRSSMSLSOYMPPLCDPKDG 1100

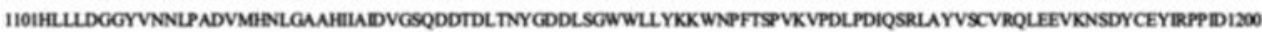
1201 KYKTLAFCSPEIRDVGYVFKNYFESMAKAGLLGFNOWFNKEYPKRVNHASLNEYTFDUAIVCLPETYAVNTAELFSEDEDCDGYISEPTTLNTD 1300 130IRRRLQVSRAGNSLSFSETEMDSDVELDUKLERKTDKSTQSSPPSNSRSDMRGKEEARHMSNWHWGVKHKDETCSCANEATKTQTCQEQELOEQQDQGATI400 D.w
}

1401 AEQLVDKDKE WNKENRSSPNNETKN 1425

Figure 2. Conceptual sequence of SWS protein with identified location of sws mutations.

Previously, two transcripts of sws gene were described [14,9]. The larger transcript SWS-RA of $5.4 \mathrm{~kb}$ is expressed at all developmental stages, prominently in young embryos and adult heads and bodies, whereas the smaller SWS-RB $1.7 \mathrm{~kb}$ transcript is only detectable in adult heads. Later, the third transcript SWS-RC of $5.4 \mathrm{~kb}$ was discovered (FlyBase). 
The products are alternatively spliced transcripts from the same transcription unit: SWS-B is the first four exons of SWS-RA; SWS-RC differs from SWS-RA only in the fourth exon. Previously identified $s w s^{1}$ and $s w s^{4}$ mutations are specific to a larger SWS-RA [9]: in $s w s^{1}$, a C to A nucleotide exchange at 1616 position produces a stop codon in place of a codon for serine, while in $s w s^{4}$, nucleotide 3357 is changed from G to A, substituting asparagine for glycine. We also have examined the ORF of SWS-RA transcript in $s w s^{76-15}$ line and determined a new, significant single nucleotide substitution. In $s w s^{76-15}$, the $G$ at position 4233 was replaced by a C, causing the substitution of glutamic acid to aspartic acid (Figure 2).

\section{Expression of SWS protein in the larval neuromusculature}

To analyze the SWS expression pattern, we used an immunohistochemical method with antisws and anti-horseradish peroxidase (HRP) (a widely used marker of neuronal membrane)
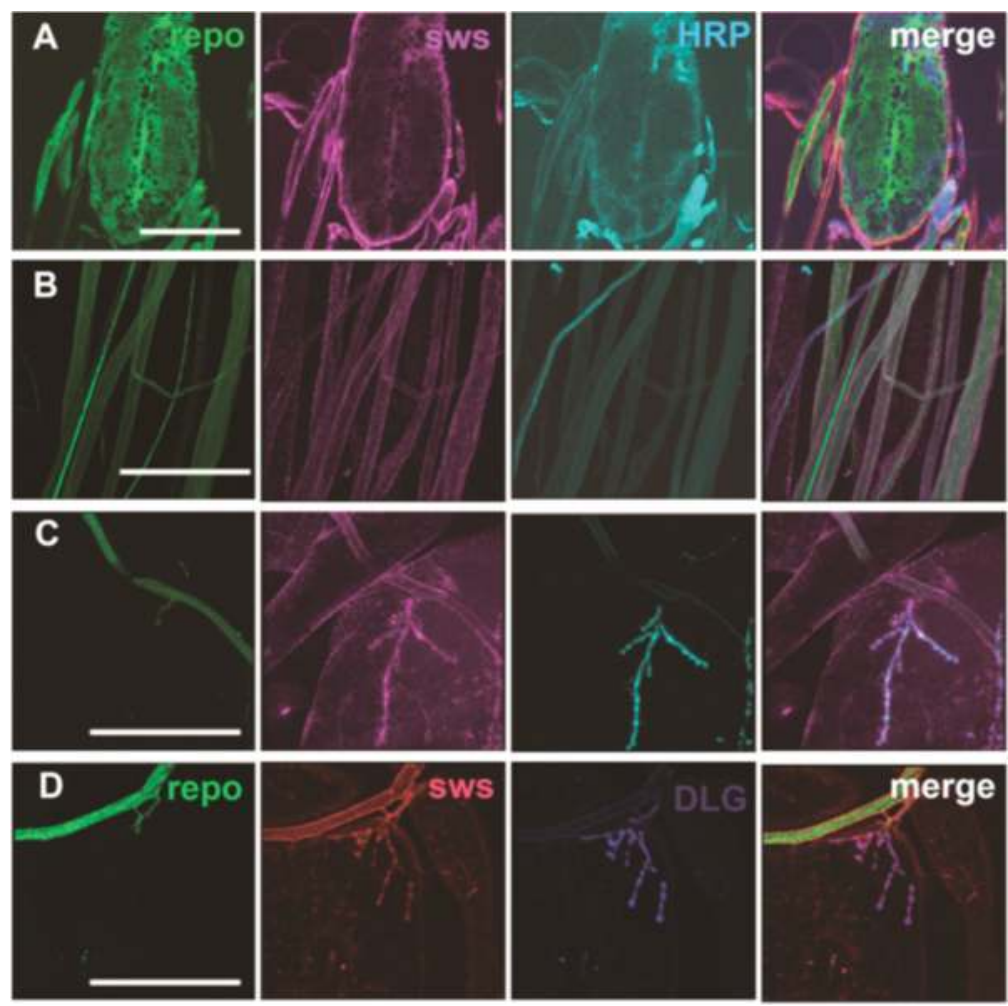

Figure 3. Distribution of SWS protein in the larval neuromusculature. SWS is localized at the presynaptic sides of larval NMJs. Figures (A-C) represent confocal imaging analysis of control Canton $S$ larval brain (A), axons (B), NMJs on muscle 4 at abdominal segments A3-A4 (C) immunohistochemically stained with repo antibody (green), SWS antibody (purple), presynaptic marker HRP (blue), and repo, SWS, and HRP merged (green, purple, and blue). Figure D represents confocal imaging analysis of SWS in larval NMJs on muscle 4 at abdominal segments A3-A4 immunohistochemically stained with repo antibody (green), SWS antibody (red), postsynaptic marker Dlg (indigo), and repo, SWS, and Dlg merged. Scale bar: $50 \mu \mathrm{m}$. 
antibodies [15]. Results showed that in wild-type larvae, SWS was expressed in cells of the ventral nerve cord (VNC) and localized in axons; here, a smaller amount of sws was found in $\mathrm{NMJ}$ in the same area as the HRP signal confirming its presynaptic localization (Figure 3A-C). To detect SWS localization in the postsynaptic region of NMJ synapses, we analyzed larvae expressing the postsynaptic density marker protein Discs Large (Dlg) [15]. Double labeling with an antibody against Dlg revealed that SWS immunoreactivity does not extend into the postsynaptic area (Figure 3D). To determine whether sws is expressed in glial cells, we used anti-glia marker-repo [16]. The analysis showed that sws is expressed in glial cells, but only those located around the brain and axons (Figure 3A-C). A similar pattern of sws expression was observed in adult $D$. melanogaster brain $[9,10]$.

\section{4. sws mutants and SWS knockdown display a changed number of satellite boutons at the NMJ}

The localization of SWS in synaptic boutons assumes its participation in NMJ development. In order to study this possible role of SWS, we used a transgenic line CD8;D42 expressing green fluorescent protein (GFP) in the neuronal membrane. The NMJs (abdominal segment 3 and muscle 4) of the offspring from CD8;D42 and SWS-RNAi and sws mutants ( $\operatorname{sws}^{1}$ and $s w s^{76-15}$ ) were analyzed using confocal microscopy [17] (Figure 4). Morphology analysis was done by the estimation of synaptic bouton number and NMJ area. The analysis showed that the NMJ

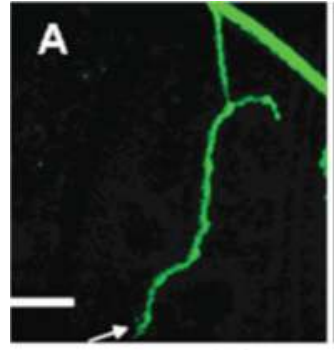

$\mathbf{E}$

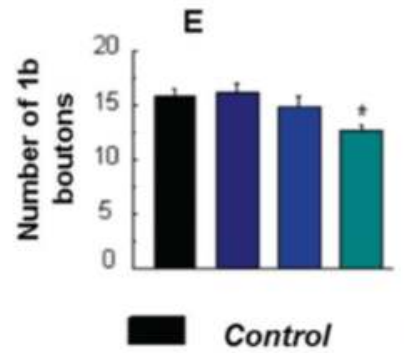

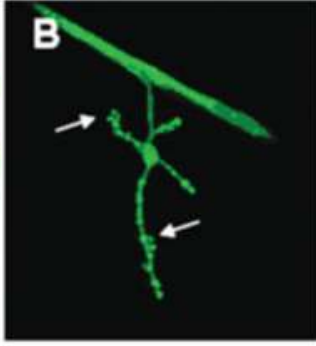

$\mathbf{F}$

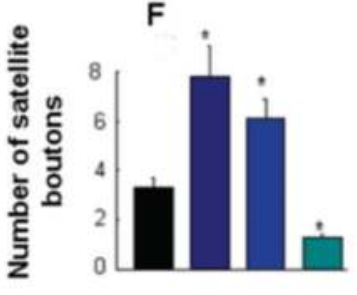

RNA $i$
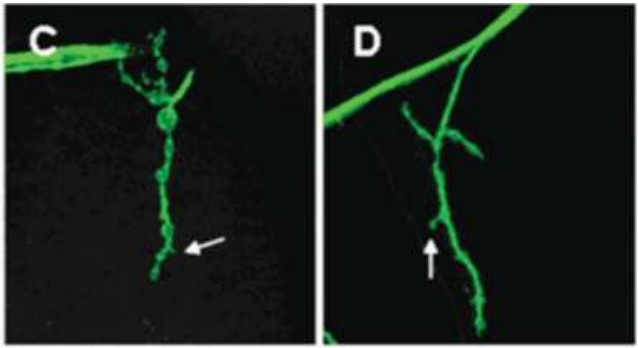

G

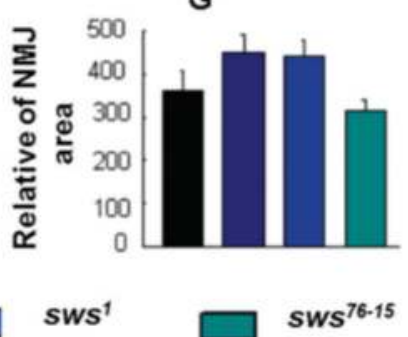

Figure 4. NMJ morphology is altered in sws mutants and SWS knockdown in motor neurons. Representative confocal images of larval NMJs (muscle 4, hemi-segments 3-4) with illuminated neuronal membrane in controls (CD8/+;D42/+) (A), SWS-RNAi//CD8/+;D42/+ (B), sws ${ }^{1} ; C D 8 /+; D 42 /+(C), s^{76-15} ; C D 8 /+; D 42 /+(D)$. Arrowheads point to satellite boutons. Bar $=25 \mu \mathrm{m}$. (E-G) Quantitative analysis of NMJ morphology in every genotype: total number of $1 \mathrm{~b}$ boutons (F) and number of satellite boutons $(\mathrm{G})$, relative area of $\mathrm{NMJs}(\mathrm{H}) .{ }^{*} \mathrm{P}<0.05$. 
area (Control-362.5 \pm 47.1 ; SWS-RNAi-448.6 \pm 39.2 ; sws $-436.7 \pm 37.1$; sws sw-15 $^{76}-314.4 \pm 25.2$, $\mathrm{p}>0.05)$ and the total number of $1 b$ boutons were not significantly different in the mutants compared to the control, except for $s w s^{76-15}$, with a slightly decreased total number of synaptic boutons (Figure $4 \mathbf{F}$ and $\mathbf{G}$ ). Interestingly, different mutations altered a number of satellite boutons (boutons budding off the parental large synaptic boutons or interboutonal space) differently (Figure 4A-D). We observed an increased number in satellite boutons in SWS-RNAi and $s w s^{1}$, but a decreased number of them in $s w s^{76-1}$ (Figure $4 \mathrm{D}$ and $\mathrm{G}$ ).

\section{The abundance of synaptic marker Bruchpilot and Dlg is altered in sws mutants and SWS knockdown}

We further examined potential defects in synaptic organization of sws mutant NMJs using synaptic markers. We analyzed presynaptic marker Bruchpilot and the postsynaptic marker Dlg in sws mutants to check whether the satellite bouton change is associated with alterations in the subcellular localization of synaptic proteins. For active zone analysis, we performed immunohistochemical staining with nc82 antibodies against a D. melanogaster-active zone protein-Bruchpilot [18]. Active zone number in SWS-RNAi and $s w s^{1}$ was not different from the control but was reduced in $s w s^{76-15}$ (Figure 5), which is, in our view, with a decrease in the number of satellite boutons. Next, we determined the number of active zones per one bouton. As seen in Figure 5, the average number of active zone contained in one bouton was significantly lower compared to that of the control (Figure 5E).
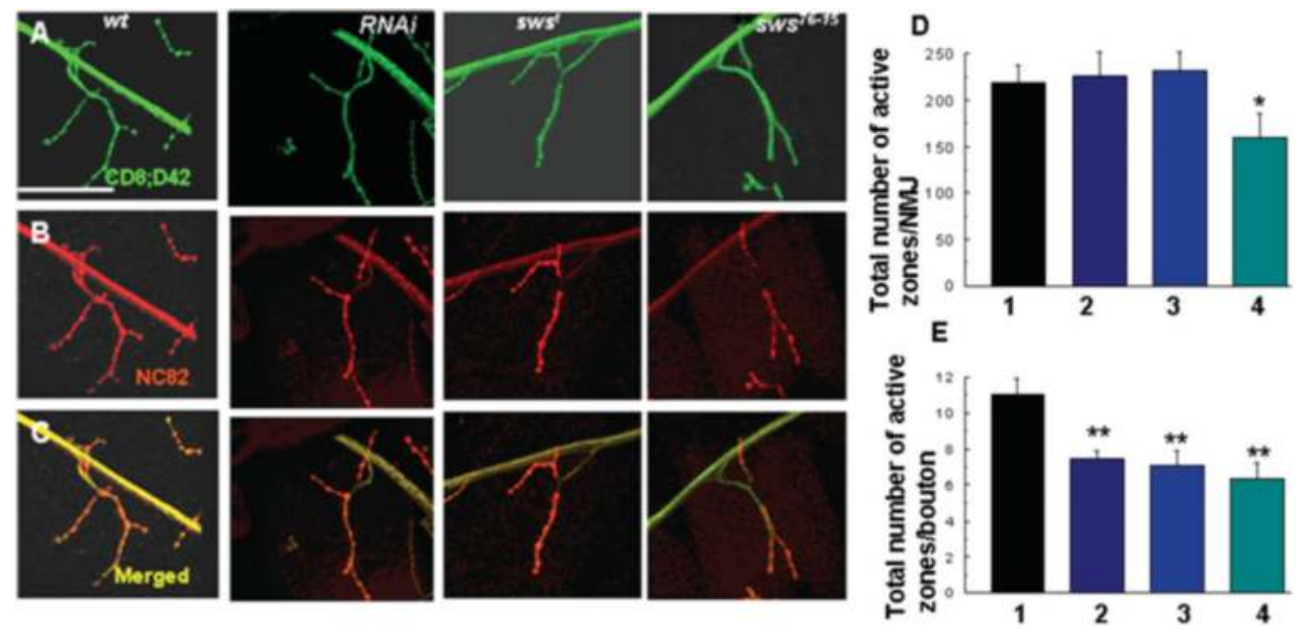

Figure 5. Distribution of active zones in SWS knockdown and sws mutants. (A-C) Representative confocal images of larval NMJs (muscle 4, hemi-segments 3-4) stained for active zones (nc82) in controls. Bar $=10 \mu \mathrm{m}$. (D-E) Quantification of the total number of active zones in a single NMJ and in a single bouton (F) in every genotype. 1, Control; 2, RNAi; 3 , sws $^{1} ; 4$, sws $^{76-15}$. ${ }^{* *} \mathrm{P}<0.01,{ }^{*} \mathrm{P}<0.05$. 

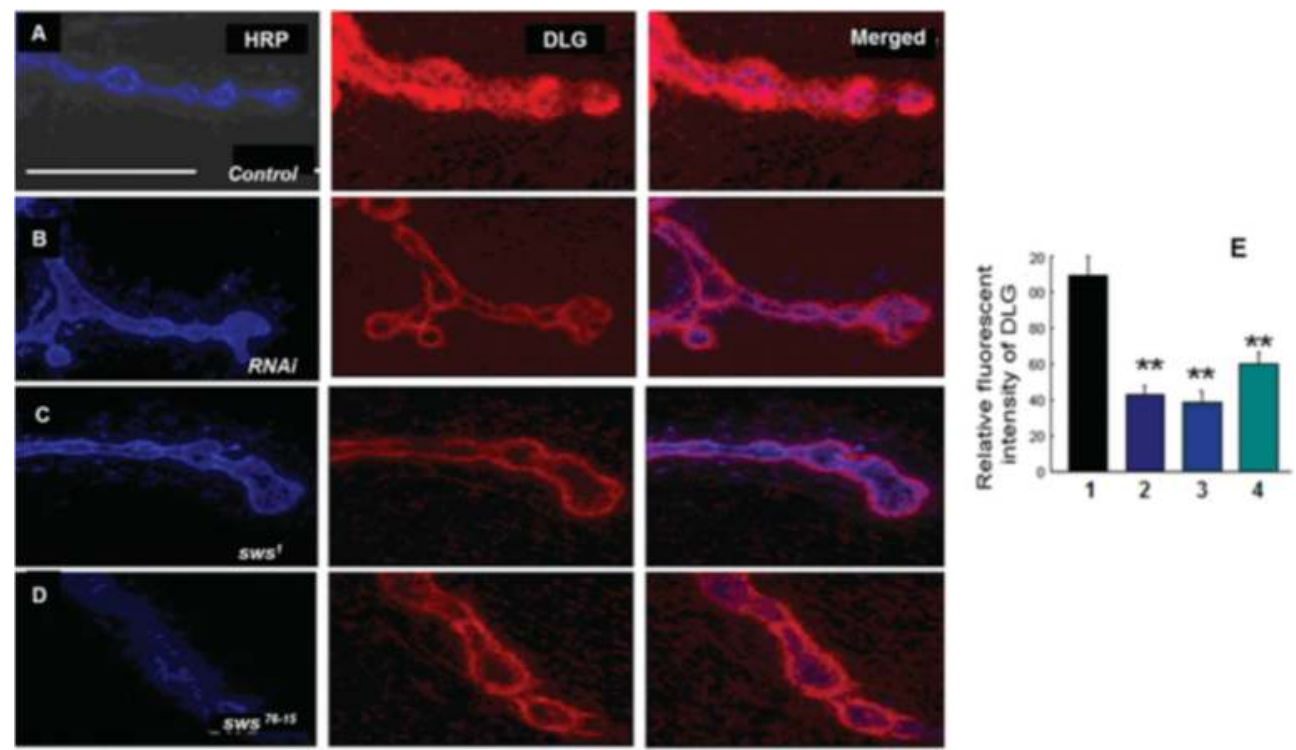

Figure 6. The synaptic levels of Dlg are reduced in SWS knockdown and sws mutants. (A-E) Representative confocal images of larval NMJs (muscle 4, hemi-segments 3-4) stained for HRP and DLG in controls (A), RNAi (B), sws ${ }^{1}$ (C), $\operatorname{sws}^{76-15}$ (D). Bar $=20 \mu \mathrm{m}$. (E) Quantification of relative fluorescent intensity of Dlg in NMJs. 1, Control; 2, RNAi; 3, sws ${ }^{1}$; $4, \operatorname{sws}^{76-15}$. "** $\mathrm{P}<0.01$.

For postsynaptic density analysis, we used antibodies against Dlg protein, the PSD 95 homo$\log$ in mammals [19]. It has been previously shown that Dlg localizes in the subsynaptic reticulum (SSR), located around $\mathrm{Ib}$ NMJ boutons, and regulates its development [20]. Anti-Dlg staining in the control line was very intensive, with a halo-like pattern around $\mathrm{Ib}$ boutons (Figure 6A), whereas the Dlg intensity level in SWS knockdown and sws mutants was significantly reduced (Figure 6B-E and F).

\section{6. sws mutants and SWS knockdown display aberrant microtubule organization}

Many synaptic bouton NMJ overgrowth phenotypes, particularly those with excess satellite boutons as well as disrupted distribution and reduced level of synaptic proteins, often cause an alteration of microtubule (MT) organization [15, 21, 22]. MT structural and dynamical regulation is one of the most important elements of synapse formation control. MTs are also necessary for synaptic terminal stabilization in synaptic development. To analyze presynaptic MTs of NMJs, we used mAb 22C10 antibodies against MT-associated neuronal protein Futsch [23]. During normal synaptic growth of the D. melanogaster NMJ, Futsch is found in association with loops of bundled microtubules typically observed within stable boutons. Loops are usually present at wild-type D. melanogaster NMJ, though in small amounts, and especially 

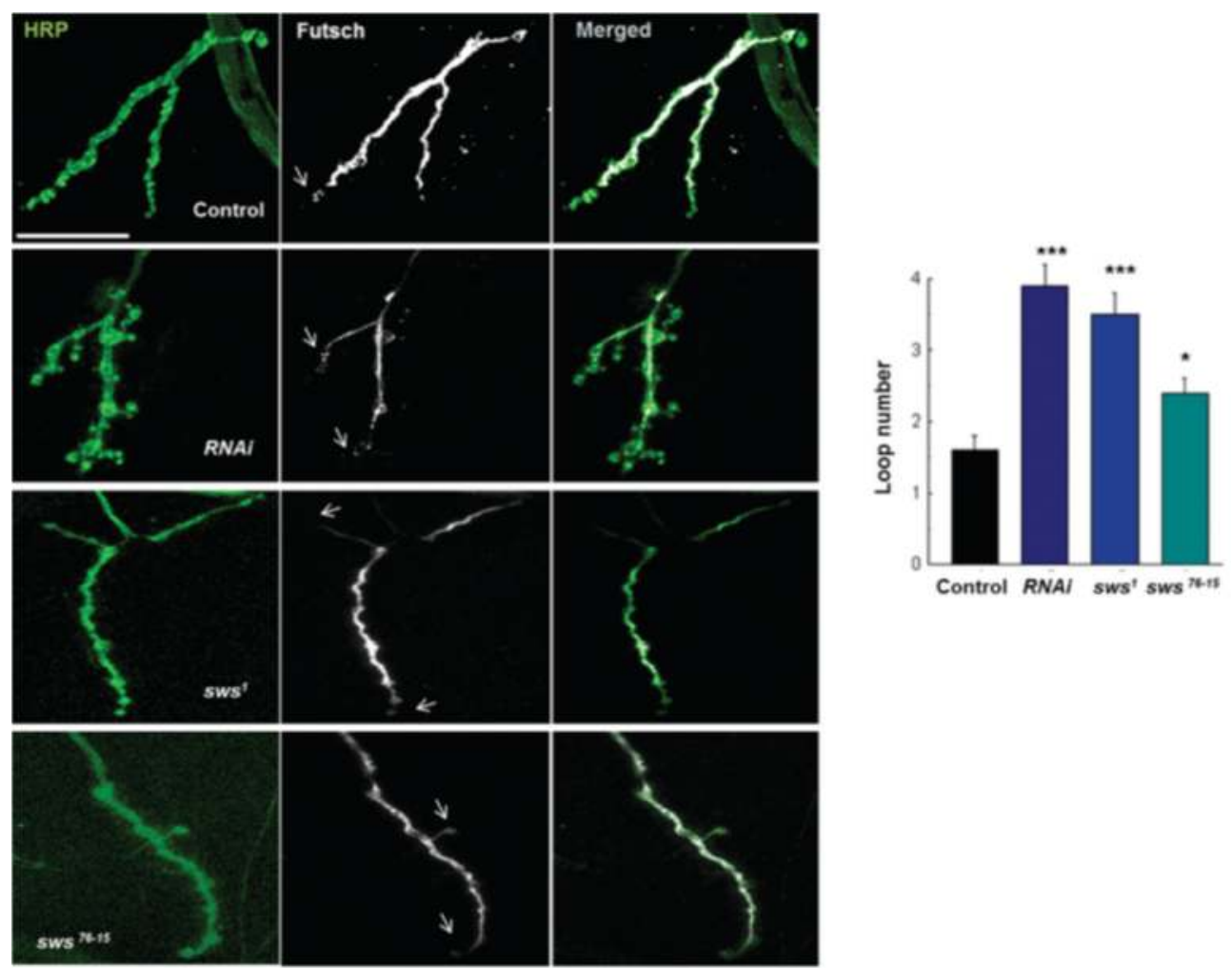

Figure 7. The number of presynaptic Futsch-positive loops is increased at synapses in SWS knockdown and sws-mutant larvae. (A-E) NMJ terminals of wild type (A), SWS-RNAi (B), sws ${ }^{1}(\mathrm{C}), s \mathrm{~s}^{76-15}(\mathrm{D})$, co-stained with anti-HRP (green) and anti-Futsch (white). Futsch-positive loops are indicated by arrowheads. Scale bar: $25 \mu \mathrm{m}$. (E) Statistical comparison of the number of Futsch-positive loops at the NMJ terminals in different genotypes. The number of NMJs analyzed for each genotype is given on the bars. ${ }^{* * * \mathrm{P}}<0.001,{ }^{*} \mathrm{P}<0.05$; error bars indicate s.e.m.

at the branch points and within terminal boutons [21]. An increase in looped MTs in the presynaptic terminal suggests MT abnormalities. Quantification of the number of Futsch-positive loops on the NMJ innervating muscle 4 showed that SWS knockdown and sws ${ }^{1}$-mutant NMJs contained significantly more number of microtubule loops compared with controls $(\mathrm{P}<0.001$; Figure 7A-C and F). Instead, within the many of mutant boutons, sws $s^{76-15}$ larvae exhibit only a slightly elevated number of MT loops at the NMJ (Figure 7D and F).

\section{7. sws mutations and SWS knockdown cause disrupted mitochondrial organization in NMJs}

Mitochondria play an important role in energy-dependent processes of synaptogenesis [24, 25], and, as shown earlier, mitochondrial transport depends on the MT maintenance [25]. In order to analyze mitochondria at NMJ synapses, we analyzed the distribution of mitochondria labeled by 

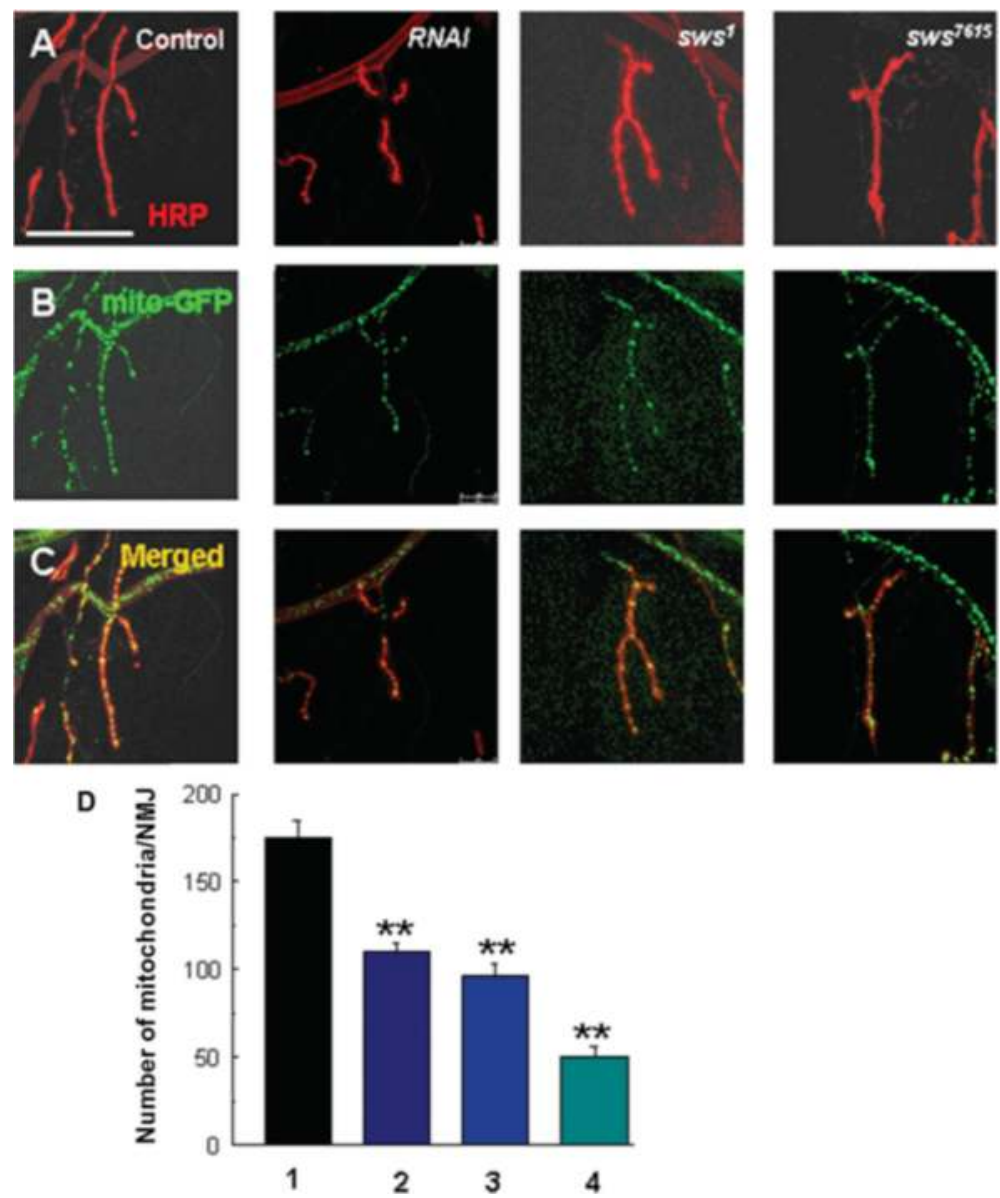

Figure 8. NMJ distribution of mitochondria in sws mutants. (A-C) Representative confocal images of larval NMJs (muscle 4, hemi-segments 3-4) visualized with mitochondria expressing GFP in controls (mito-GFP/+;D42/+). Bar $=10 \mu \mathrm{m}$. (D) Quantification of mitochondria in NMJs in every genotype. 1, Control; 2, RNAi; 3, sws ; 4, sws ${ }^{76-15}$. ${ }^{*} \mathrm{P}<0.01$.

a green fluorescent protein (GFP) tag (mito-GFP) in control strain and sws mutants. The mito-GFP tag was constructed by fusing the N-terminal 31 amino acid (mitochondria-targeting) sequence from human cytochrome C oxidase subunit VIII with N-terminus of GFP [26]. Mitochondria expressing GFP are very easy to observe in NMJs of control motor neurons (Figure 8A). However, the number of mitochondria was significantly reduced in mutant lines compared to that in control (Figure 8A-D and E).

\section{Summary and concluding remarks}

In this chapter, we investigated the role of sws in the development and functioning of the nervous system using an excellent well-developed model of D. melanogaster larval NMJs. We showed 
that sws is widely expressed in the larval nervous system and localized in the brain, axons, and NMJs. However, SWS is predominantly found in glial cells of the brain and axons. These data correlate with the sws distribution in the brain of adult flies [10,27]. We also described a new mutation in the sws gene $\left(s w s^{76-15}\right)$ and compared its phenotypic manifestations in the nervous system of the larvae to the previously described $\operatorname{sws}^{1}$ mutation and SWS knockdown. We show the presynaptic depletion of SWS in the motor neurons using GAL4-inducible fly line SWS$R N A i$, and null sws ${ }^{1}$ mutations result in a significant expansion of synaptic bouton number. Interestingly, the number of $1 \mathrm{~b}$ and satellite boutons in the $s w s^{76-15}$ mutant was decreased in the mutant as compared with that in the control. These results indicate that SWS controls synaptic bouton formation and differentiation of the NMJ during the third-instar larval stage.

Satellite bouton production has been observed for certain D. melanogaster proteins Spastin [28] and Atlastin [29] implicated in HSP. How mutations in sws lead to the formation of excess or deficiency satellite boutons remains to be elucidated. However, as previously shown, mutants characterized by extra satellite boutons can display changing the number of microtubule loops [15]. We found that SWS knockdown $s w s^{1}$ and $s w s^{76-15}$ mutations resulted in more stable MT loops in the NMJ. One of our discoveries is that sws mutations cause mitochondrial dysfunction in NMJs, which is a common effect in other HSP forms [30-32]. Mitochondrial transport to synapse is tightly regulated to provide sufficient energy for synaptic transmission $[33,34]$. Decreased synaptic transmission has been reported to be associated with a reduced number of functional mitochondria [35, 36]. Indeed, glial SWS knockdown induces defects in neuronal transmission; however, levels of Bruchpilot, an active-site marker, were unchanged [26]. Here, we show that although there was an increase in bouton number at NMJs of sws mutants, the boutons did not show a reduction in the abundance in the active zone synaptic function marker Bruchpilot, while their number was significantly lower in the single bouton, suggesting a decrease in their neurotransmitter function.

Six alleles of the D. melanogaster sws gene were described, and two of these, sws ${ }^{1}$ and $s w s^{5}$, mutations and SWS knockdown were characterized at the biochemical level $[9,37,26] . \operatorname{sws}^{1}$ is the most completely studied sws mutation and results in a truncated protein of about a fourth of the original length [9]. Previous reports on sws mutants have described abnormal morphology only in the brain and eye of the adult fly, but have correlated these changes with the loss of SWS catalytic activity $[9,10]$. Novel point mutant, $s w s^{76-15}$, is located in the region of SWS, which has no homology with NTE. On this basis, we believe that the mutant has not broken esterase function. In our study, SWS knockdown $s w s^{1}$ and $s w s^{76-15}$ acted practically identically, which shows, for our point of view, that we revealed the functions of SWS, which are not connected with esterase

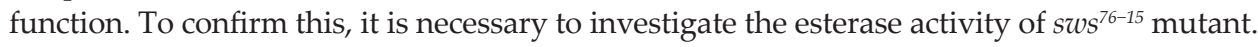

When discussing about SWS functions in the nervous system, we should remember the fact that the most prominent expression sws was found in glial cells on the surface of the brain and axons. Indeed, a recent study showed that the loss of SWS in glia impairs neuronal function, strongly suggesting that the loss of glial SWS plays an important role in the phenotypes observed in the sws mutant [26]. We did not observe significant and gross changes in glia morphology, presumably because of the short developmental larval stage. 
In summary, our research showed the role of sws in the regulation of NMJ functioning. Further studies about processes regulated by sws will help us to better understand the molecular mechanisms underlying the pathogenesis of diseases.

\section{Materials and methods}

\subsection{D. melanogaster lines}

Ethylmethane sulfonate (EMS)-induced mutant alleles of sws were used as a research material and wild-type Oregon $R$ line as a control. All mutants were isolated in screens for structural brain defects using the histology brain assay [38]: $s w s^{1}$ is previously described by Kretzschmar et al. [9], $s w s^{76-15}$ (also referred as 76-15 line) isolated by Shcherbata et al. [13]. Another strain was obtained from the Bloomington Drosophila Stock Centre (Indiana University, USA). All strains were kept on standard a medium at $25^{\circ} \mathrm{C}$.

\subsection{Tissue sections}

\subsubsection{Paraffin sections}

The mass histology procedure by Heisenberg and Bohl [38] was used for general neurodegenerative phenotype analysis in adult flies. Flies were placed into collars and fixed in Carnoy solution (ethanol-chloroform-acetic acid, 6:3:1) at $4^{\circ} \mathrm{C}$, which was followed by their dehydration in ethanol (30 min), methyl benzoate $(30 \mathrm{~min})$, and twice in paraffin (60 min). Paraffin blocks were used to prepare 7- $\mu \mathrm{m}$ thick sections. Paraffin slices were washed with xylene and covered with DPX ("Fluka," USA). The preparations were examined on a Carl Zeiss Jena microscope at $12 \times 40$ magnifications in UV light for eye auto-fluorescence (no staining). In the experiment, we tested at least 25 flies (20-22 days old) of each genotype.

\subsubsection{Semi-thin plastic sections}

Fly heads were dissected on ice and fixed in $2 \%$ glutaraldehyde and $2 \%$ osmium solutions for $6 \mathrm{~h}$. After removing osmium, the heads were dehydrated in ethanol solutions of increasing concentrations (30,50,70, 90, and 100\%) and subsequently incubated two times for 2 min in propylene oxide solution at room temperature. Afterwards, the propylene oxide was replaced with a propylene oxide-resin mixture and left to incubate overnight. The mixture was replaced with pure resin, and the heads were incubated for three more hours. Molds for block preparation were filled with resin, and the heads were separately placed in the molds with proper orientation. Molds with oriented heads were left overnight at $70^{\circ} \mathrm{C}$ for the resin in the blocks to solidify. Then, blocks were cracked from molds and placed into signed tubes, with the subsequent preparation of $1-\mu \mathrm{m}$ thick sections using a semiautomatic Historange Microtome using a diamond knife. Sections were stained with the Toluidine Blue solution, washed with distilled water, and covered with glass using DPX-mountant for histology [38]. 


\subsection{Genetic analysis of $s w s^{76-15}$}

\subsubsection{Mapping}

Complementation analysis includes obtaining of trans-heterozygotes to control recessive mutations of the same trait. Mapping was performed by crossing mutants with a deficiency line (Df (1) C128/FM6) and a line with duplication (Df(1)ct-J4, In(1)dl-49, f(1)/C(1)DX, y(1) $\mathrm{w}(1) \mathrm{f}(1) ; \mathrm{Dp}(1 ; 3) \operatorname{sn}(13 \mathrm{a} 1) /+)$ in the same band of 7D1 X-chromosome. Both lines were kindly provided by the Bloomington Stock Centre.

\subsubsection{Molecular identification}

Total RNA was extracted from 22-day-old fly heads using TRIZOL LS ("Life Technologies," Switzerland) according to a standard technique [37]. The extracted RNA was dissolved in $\mathrm{MQH}_{2} \mathrm{O}$ with $0.1 \%$ DEPC (diethyl pyrocarbonate) and stored at $-80^{\circ} \mathrm{C}$. Using the Primer Select software, we developed eight pairs of primers to the ORF SWS-RA transcript (4274 bp) sequence. Expected fragments were from 620 to $829 \mathrm{bp}$ and overlapped with each other. The cDNA was synthesized using RNaseOUT Recombinant Ribonuclease Inhibitor and Super Script II RNase H-Reverse Transcriptase ("Invitrogen," USA). cDNA was used as a template in polymerase chain reaction (PCR) with Expand High-Fidelity PCR System ("Roche Diagnostics," Germany). After polymerization, $0.5 \mathrm{ml}$ Taq-polymerase ("Roche Diagnostics," Germany) and $0.5 \mathrm{ml} \mathrm{dATF}$ were added to the mixture and incubated for $30 \mathrm{~min}$ at $72^{\circ} \mathrm{C}$ to form the polyadenine tail for subsequent T/A cloning in pGEM-T-Easy Vector. The size of the fragments was estimated by their electrophoretic movement in $1 \%$ agarose gel compared to $1 \mathrm{~kb}$ Plus DNA Ladder. DNA fragments were eluted from gel using GFP PCR DNA and Gel Band Purification Kit ("Amersham Biosciences," GB). DNA sequencing was performed by Macrogen Inc. (Korea); each fragment from PCR of different clones was tested three times in both directions. Using the DNA Star software, we performed comparative analysis between the cDNA fragments of sws gene nucleotide sequence from the Gene Bank database (NCBI) in wild-type strain Oregon- $R$ and $s w s^{76-15}$ mutant.

\subsection{Sample preparation and assay of neuromuscular junction morphology}

The third-stage larvae were dissected in freshly prepared $\mathrm{HL} 3$ solution $(110 \mathrm{mM} \mathrm{NaCl}, 5 \mathrm{mM} \mathrm{KCl}$, $10 \mathrm{mM} \mathrm{NaHCO}_{3}, 5 \mathrm{mM} \mathrm{HEPES}, 30 \mathrm{mM}$ sucrose, $5 \mathrm{mM}$ trehalose, $10 \mathrm{mM} \mathrm{MgCl}{ }_{2}$ pH 7.2) (Budnik and Ruiz-Canada, 2006). Then, they were fixed with $4 \%$ formaldehyde (Sigma-Aldrich, United States) for $15 \mathrm{~min}$, washed with phosphate buffer saline (PBS) and mounted in VectaShield mounting medium (Vector Laboratories, USA). Samples were analyzed with Leica TCS-SP5 laser confocal microscope (Leica, Germany). Bouton number, axon branch number, and neuromuscular junction length were estimated with the ImageJ software (http://imagej.nih.gov/ij/) and LAS AF Lite software (Leica, Germany). Then, 6-8 larvae of each genotype were analyzed. Each experiment was done in triplicate. For comparison between genotypes, all samples were processed simultaneously and imaged using identical microscopic acquisition parameters. All images were also corrected for any background before any intensity measurements. 


\subsection{Mitochondria assay}

Larvae were dissected in HL3, fixed with $4 \%$ paraformaldehyde for $15 \mathrm{~min}$, washed with PBS ( $3 \times 15 \mathrm{~min})$, and mounted in VectaShield mounting medium (Vector Laboratories, USA). Samples were analyzed using a Leica TCS-SP5 laser confocal microscope (Leica, Germany) at $488 \mathrm{~nm}$. Relative fluorescence was estimated using the ImageJ software. In total, 6-8 larvae of each genotype were analyzed. Each experiment was performed in triplicate.

\subsection{Immunohistochemistry}

Third-instar larvae were dissected in PBS, fixed in 4\% paraformaldehyde for $20 \mathrm{~min}$, and washed with PBS $(3 \times 15 \mathrm{~min})$. Then, larvae were blocked in blocking buffer BlockPRO (Visual Protein Biotechnology Corporation, USA) for $1 \mathrm{~h}$ at room temperature, followed by overnight incubation at $4^{\circ} \mathrm{C}$ in primary antibodies (diluted in BlockPRO) and washing in PBS $(3 \times 15 \mathrm{~min})$. Afterward, larvae were incubated in secondary antibodies (diluted in BlockPRO) for $2 \mathrm{~h}$, followed by washing in PBS $(3 \times 15 \mathrm{~min})$ and mounting in VectaShield medium (Vector Laboratories, USA). We used the following primary antibodies: rabbit anti-sws (1:100; Almabion, Russia), mouse anti-Brp (Bruchpilot) (1:200; mAb NC82; Developmental Studies Hybridoma Bank (DSHB), USA), mouse anti-Dlg ((1:200; mAb 4F3; Developmental Studies Hybridoma Bank (DSHB), USA), mouse anti-Futsch ((1:200; Developmental Studies Hybridoma Bank (DSHB), USA), and secondary antibodies: goat anti-mouse Cy3-conjugated (1:400, Jackson ImmunoResearch, USA). Antibodies were obtained from the Developmental Studies Hybridoma Bank developed under the auspices of the NICHD and maintained by The University of Iowa, Department of Biology, Iowa City, IA.

\subsection{Statistics}

Statistical analyses were performed using the KyPlot software (KyensLab Inc.). A one-way analysis of variance (ANOVA) was followed by planned multiple comparisons between relevant groups with Tukey-Kramer test.

\section{Acknowledgements}

We thank Dr. Doris Kretzschmar for providing anti-sws antibody vector. We also thank Prof. Christos Samakovlis and Prof. Karl-Friedrich Fischbach for insight, discussion, advice, and support.

\section{Conflict of interest statement}

The authors have declared that no competing interests exist. 


\section{Funding}

This work was supported by the Russian Fund for Basic Research (15-04-09041 to S.S.), INTAS (04-83-3248 to N.M.), and DAAD fellowship for young scientists to I.M.

\section{Author details}

Elena Ryabova ${ }^{1}$, Nataliya Matiytsiv², Olena Trush², Iryna Mohylyak², Galina Kislik ${ }^{1}$, Pavel Melentev ${ }^{1}$ and Svetlana Sarantseva ${ }^{1 *}$

*Address all correspondence to: svesar1@yandex.ru

1 B.P. Konstantinov Petersburg Nuclear Physics Institute, National Research Centre

"Kurchatov Institute", Gatchina, Russia

2 Department of Genetics and Biotechnology, Ivan Franko National University of Lviv, Lviv, Ukraine

\section{References}

[1] Rainier S, Bui M, Mark E, Thomas D, Tokarz D, Ming L, Delaney C, Richardson RJ, Albers JW, Matsunami N, Stevens J, Coon H, Leppert M, Fink JK. Neuropathy target esterase gene mutations cause motor neuron disease. American Journal of Human Genetics. 2008;82:780-785. DOI: 10.1016/j.ajhg.2007.12.018

[2] Synofzik M, Gonzalez MA, Lourenco CM, Coutelier M, Haack TB, Rebelo A, Hannequin D, Strom TM, Prokisch H, Kernstock C, Durr A, Schöls L, Lima-Martínez MM, Farooq A, Schüle R, Stevanin G, Marques W Jr, Züchner S. PNPLA6 mutations cause BoucherNeuhäuser and Gordon Holmes syndromes as part of a broad neurodegenerative spectrum. Brain. 2014;137(Pt 1):69-77. DOI: 10.1093/brain/awt326

[3] Hufnagel RB, Arno G, Hein ND, Hersheson J, Prasad M, Anderson Y, Krueger LA, Gregory LC, Stoetzel C, Jaworek TJ, Hull S, Li A, Plagnol V, Willen CM, Morgan TM, Prows CA, Hegde RS, Riazuddin S, Grabowski GA, Richardson RJ, Dieterich K, Huang T, Revesz T, Martinez-Barbera JP, Sisk RA, Jefferies C, Houlden H, Dattani MT, Fink JK, Dollfus H, Moore AT, Ahmed ZM. Neuropathy target esterase impairments cause OliverMcFarlane and Laurence-Moon syndromes. Journal of Medical Genetics. 2015;52:85-94. DOI: 10.1136/jmedgenet-2014-102856

[4] Kmoch S, Majewski J, Ramamurthy V, Cao S, Fahiminiya S, Ren H, MacDonald IM, Lopez I, Sun V, Keser V, Khan A, Stránecký V, Hartmannová H, Přistoupilová A, Hodaňová K, Piherová L, Kuchař L, Baxová A, Chen R, Barsottini OG, Pyle A, Griffin H, Splitt M, Sallum J, Tolmie JL, Sampson JR, Chinnery P, Care4Rare Canada, Banin E, Sharon D, Dutta S, Grebler R, Helfrich-Foerster C, Pedroso JL, Kretzschmar D, Cayouette M, Koenekoop RK. Mutations in PNPLA6 are linked to photoreceptor degeneration and various forms of childhood blindness. Nature Communications. 2015;6:5614. DOI: 10. 1038/ncomms6614 
[5] Johnson MK. The delayed neurotoxic action of some organophosphorus compounds. Identification of the phosphorylation site as an esterase. The Biochemical Journal. 1969; 114:11-17

[6] Glynn P. A mechanism for organophosphate-induced delayed neuropathy. Toxicology Letters. 2006;162:94-97. DOI: 10.1016/j.toxlet.2005.10.012

[7] Fink JK. Hereditary spastic paraplegia. Current Neurology and Neuroscience Reports. 2006;6:65-76. DOI: 10.1055/s-0034-1386767

[8] Moser M, Stempfl T, Li Y, Glynn P, Büttner R, Kretzschmar D. Cloning and expression of the murine sws/NTE gene. Mechanisms of Development. 2000;90:279-282

[9] Kretzschmar D, Hasan G, Sharma S, Heisenberg M, Benzer S. The Swiss cheese mutant cause glial hyperwrapping and brain degeneration in Drosophila. The Journal of Neuroscience. 1997;17:7425-7432

[10] Mühlig-Versen M, da Cruz AB, Tschäpe JA, Moser M, Büttner R, Athenstaedt K, Glynn P, Kretzschmar D. Loss of Swiss cheese/neuropathy target esterase activity causes disruption of phosphatidylcholine homeostasis and neuronal and glial death in adult Drosophila. The Journal of Neuroscience. 2005;25:2865-2873. DOI: 10.1523/JNEUROSCI.5097-04. 2005

[11] Akassoglou K, Malester B, Xu J, Tessarollo L, Rosenbluth J, Chao MV. Brain-specific deletion of neuropathy target esterase/Swiss cheese results in neurodegeneration. Proceedings of the National Academy of Sciences of the United States of America. 2004;101:5075-5080. DOI: $10.1073 /$ pnas.0401030101

[12] Song Y, Wang M, Mao F, Shao M, Zhao B, Song Z, et al. Knockdown of Pnpla6 protein results in motor neuron defects in zebra fish. Disease Models \& Mechanisms. 2013;6: 404-413. DOI: $10.1242 / \mathrm{dmm} .009688$

[13] Shcherbata GR, Matiŭtsiv NP, Chernik IAI, Iatsenko AS, Radysh VV, Kucherenko MM, Maksimiv DV. Genetic analysis of X-chromosome Drosophila melanogaster neurodegenerative mutants induced by ethylmethanesulphonate and nitrosoethylurea. Genetika. 2004;40:1286-1292

[14] Hasan G. Molecular cloning of an olfactory gene from Drosophila melanogaster. Proceedings of the National Academy of Sciences of the United States of America. 1990;87:9037-9041

[15] Franco B, Bogdanik L, Bobinnec Y, Debec A, Bockaert J, Parmentier ML, Grau Y. Shaggy, the homolog of glycogen synthase kinase 3, controls neuromuscular junction growth in Drosophila. The Journal of Neuroscience. 2004;24:6573-6577. DOI: 10.1523/JNEUROSCI. 1580-04.2004

[16] Xiong WC, Okano H, Patel NH, Blendy JA, Montell C. Repo encodes a glial-specific homeo domain protein required in the Drosophila nervous system. Genes \& Development. 1994; 8:981-994

[17] Budnik V, Ruiz-Canada C. The Fly Neuromuscular Junction: Structure and Function. Int. Rev. Neurobiol. 2nd ed. San Diego: Academic Press; 2006 
[18] Wagh DA, Rasse TM, Asan E, Hofbauer A, Schwenkert I, Dürrbeck H, Buchner S, Dabauvalle MC, Schmidt M, Qin G, Wichmann C, Kittel R, Sigrist SJ, Buchner E. Bruchpilot, a protein with homology to ELKS/CAST, is required for structural integrity and function of synaptic active zones in Drosophila. Neuron. 2006;49:833-844. DOI: 10.1016/j.neuron.2006.02.008

[19] Cohen RS, Blomberg F, Berzins K, Siekevitz P. The structure of postsynaptic densities isolated from dog cerebral cortex. I. Overall morphology and protein composition. The Journal of Cell Biology. 1997;74:181-203

[20] Budnik V, Koh YH, Guan B, Hartmann B, Hough C, Woods D, Gorczyca M, Budnik V, Koh YH, Guan B, Hartmann B, Hough C, Woods D, et al. Regulation of synapse structure and function by the Drosophila tumor suppressor gene dlg. Neuron. 1996;17:627-640

[21] Roos J, Hummel T, Ng N, Klämbt C, Davis GW. Drosophila Futsch regulates synaptic microtubule organization and is necessary for synaptic growth. Neuron. 2000;26:371-382

[22] Viquez NM, Li CR, Wairkar YP, DiAntonio A. The B' protein phosphatase 2A regulatory subunit well-rounded regulates synaptic growth and cytoskeletal stability at the Drosophila neuromuscular junction. The Journal of Neuroscience. 2006;26:9293-9303. DOI: 10.1523/JNEUROSCI.1740-06.2006

[23] Hummel T, Krukkert K, Roos J, Davis G, Klambt C. Drosophila Futsch/22C10 is a MAP1Blike protein required for dendritic and axonal development. Neuron. 2000;26:357-370

[24] Zenisek D, Matthews G. The role of mitochondria in presynaptic calcium handling at a ribbon synapse. Neuron. 2000;25:229-237

[25] Saxton WM, Hollenbeck PJ. The axonal transport of mitochondria. Journal of Cell Science. 2012;125(Pt 9):2095-2104. DOI: 10.1242/jcs.053850

[26] Pilling AD, Horiuchi D, Lively CM, Saxton WM. Kinesin-1 and dynein are the primary motors for fast transport of mitochondria in Drosophila motor axons. Molecular Biology of the Cell. 2006;17:2057-2068. DOI: 10.1091/mbc.E05-06-0526

[27] Dutta S, Rieche F, Eckl N, Duch C, Kretzschmar D. Glial expression of Swiss cheese (SWS), the Drosophila orthologue of neuropathy target esterase (NTE), is required for neuronal ensheathment and function. Disease Models \& Mechanisms. 2016;9:283-294. DOI: $10.1242 / \mathrm{dmm} .022236$

[28] Sherwood NT, Sun Q, Xue M, Zhang B, Zinn K. Drosophila spastin regulates synaptic microtubule networks and is required for normal motor function. PLoS Biology. 2004;2:e429. DOI: 10.1371/journal.pbio.0020429

[29] Lee M, Paik SK, Lee MJ, Kim YJ, Kim S, Nahm M, SJ O, Kim HM, Yim J, Lee CJ, Bae YC, Lee S. Drosophila Atlastin regulates the stability of muscle microtubules and is required for synapse development. Developmental Biology. 2009;330:250-262. DOI: 10.1016/j. ydbio.2009.03.019 
[30] Lu J, Rashid F, Byrne PC. The hereditary spastic paraplegia protein spartin localises to mitochondria. Journal of Neurochemistry. 2006;98:1908-1919. DOI: 10.1111/j.1471-4159. 2006.04008.x

[31] Goizet C, Depienne C, Benard G, Boukhris A, Mundwiller E, Solé G, Coupry I, Pilliod J, Martin-Négrier ML, Fedirko E, Forlani S, Cazeneuve C, Hannequin D, Charles P, Feki I, Pinel JF, Ouvrard-Hernandez AM, Lyonnet S, Ollagnon-Roman E, Yaouanq J, Toutain A, Dussert C, Fontaine B, Leguern E, Lacombe D, Durr A, Rossignol R, Brice A, Stevanin G. REEP1 mutations in SPG31: Frequency, mutational spectrum, and potential association with mitochondrial morpho-functional dysfunction. Human Mutation. 2011;32:1118-1127. DOI: 10.1002/humu.21542

[32] Verny C, Guegen N, Desquiret V, Chevrollier A, Prundean A, Dubas F, Cassereau J, Ferre M, Amati-Bonneau P, Bonneau D, Reynier P, Procaccio V. Hereditary spastic paraplegia-like disorder due to a mitochondrial ATP6 gene point mutation. Mitochondrion. 2011;11:70-75. DOI: 10.1016/j.mito.2010.07.006

[33] Ly CV, Verstreken P. Mitochondria at the synapse. The Neuroscientist. 2006;12:291-299. DOI: $10.1177 / 1073858406287661$

[34] Wang X, Schwarz TL. Imaging axonal transport of mitochondria. Methods in Enzymology. 2009;457:319-333. DOI: 10.1016/S0076-6879(09)05018-6

[35] Goldstein AY, Wang X, Schwarz TL. Axonal transport and the delivery of pre-synaptic components. Current Opinion in Neurobiology. 2008;18:495-503. DOI: 10.1016/j.conb. 2008.10.003

[36] Verstreken P, Ly CV, Venken KJ, Koh TW, Zhou Y, Bellen HJ. Synaptic mitochondria are critical for mobilization of reserve pool vesicles at drosophila neuromuscular junctions. Neuron. 2005;47:365-378. DOI: 10.1016/j.neuron.2005.06.018

[37] Sujkowski A, Rainier S, Fink JK, Wessells RJ. Delayed induction of human NTE (PNPLA6) rescues neurodegeneration and mobility defects of Drosophila swiss cheese (sws) mutants. PLoS One. 2015;10:e0145356. DOI: 10.1371/journal.pone.0145356

[38] Heisenberg M, Bohl K. Isolation of anatomical brain mutants of Drosophila by histological means. Naturforsch. 1979;34:143-147 
